PROCEEDINGS OF THE

AMERICAN MATHEMATICAL SOCIETY

Volume 132, Number 8 , Pages $2483-2493$

S 0002-9939(04)07465-9

Article electronically published on March 3, 2004

\title{
CONVERGENCE OF HARMONIC MAPS ON THE POINCARÉ DISK
}

\author{
GUOWU YAO
}

(Communicated by Juha M. Heinonen)

\begin{abstract}
Let $\left\{f_{n}: \mathbb{D} \rightarrow \mathbb{D}\right\}$ be a sequence of locally quasiconformal harmonic maps on the unit disk $\mathbb{D}$ with respect to the Poincaré metric. Suppose that the energy densities of $f_{n}$ are uniformly bounded from below by a positive constant and locally uniformly bounded from above. Then there is a subsequence of $\left\{f_{n}\right\}$ that locally uniformly converges on $\mathbb{D}$, and the limit function is either a locally quasiconformal harmonic map of the Poincaré disk or a constant. Especially, if the limit function is not a constant, the subsequence can be chosen to satisfy some stronger conditions. As an application, it is proved that every point of the space $T_{0}(\mathbb{D})$, a subspace of the universal Teichmüller space, can be represented by a quasiconformal harmonic map that is an asymptotic hyperbolic isometry.
\end{abstract}

\section{INTRODUCTION}

Harmonic maps and their Hopf differentials on the Poincaré disk have been extensively studied (for example, see [1], 16]). Some relations between quasiconformal harmonic diffeomorphism and the universal Teichmüller space have also been found (cf. [15], 17]). Usually, we make the restriction that harmonic maps are quasiconformal or have finite total energy [2]. Our purpose in this paper is to study the normality of a sequence of harmonic maps on the Poincaré disk under weaker assumptions on the energy densities.

Let $\mathbb{D}$ be the unit disk $\{|z|<1\}$ with the Poincaré metric $\rho(z)|d z|^{2}$, where

$$
\rho(z)|d z|^{2}=\frac{4}{\left(1-|z|^{2}\right)^{2}}|d z|^{2}, \quad \forall z \in \mathbb{D} .
$$

In this paper, we only treat the (locally) quasiconformal harmonic maps of $\mathbb{D}$ onto itself with respect to the Poincaré metric. We shall restrict ourselves to the case where a map $f: \mathbb{D} \rightarrow \mathbb{D}$ is called a harmonic map if it is a $C^{2}$-homeomorphism of $\mathbb{D}$ onto itself and its Hopf differential $\Phi=\phi_{f}(z) d z^{2}=\rho(f(z)) f_{z} \bar{f}_{z} d z^{2}$ is holomorphic in $\mathbb{D}$.

Received by the editors May 17, 2002 and, in revised form, May 15, 2003.

2000 Mathematics Subject Classification. Primary 58E20; Secondary 30C62.

Key words and phrases. Harmonic map, energy density, locally quasiconformal map, asymptotic hyperbolic isometry.

This research was supported by the "973" Project Foundation of China (Grant No. TG199075105) and the Foundation for Doctoral Programme. 
Let $w=f(z)$ be a harmonic homeomorphism of $\mathbb{D}$ onto itself. Then it satisfies the Euler-Lagrange equation

$$
f_{z \bar{z}}+\partial_{w}(\log \rho) \circ f \cdot f_{z} f_{\bar{z}}=0 .
$$

The $\partial$ and $\bar{\partial}$ energy densities are given by

$$
|\partial w|^{2}=\frac{\rho(w)}{\rho(z)}\left|w_{z}\right|^{2}, \quad|\bar{\partial} w|^{2}=\frac{\rho(w)}{\rho(z)}\left|w_{\bar{z}}\right|^{2} .
$$

Since the curvature of the Poincaré metric is -1 , the Euler-Lagrange equation gives the following equations [12]:

$$
\begin{aligned}
& \Delta_{\rho} \log |\partial w|^{2}=2|\partial w|^{2}-2|\bar{\partial} w|^{2}-2, \\
& \Delta_{\rho} \log |\bar{\partial} w|^{2}=-2|\partial w|^{2}+2|\bar{\partial} w|^{2}-2,
\end{aligned}
$$

where $\Delta_{\rho}=\frac{\Delta_{0}}{\rho}$ and $\Delta_{0}$ is the Euclidean Laplacian.

Definition 1. A map $f: \mathbb{D} \rightarrow \mathbb{D}$ is called a locally quasiconformal map if for any subdomain $E$ with $\bar{E} \subset \mathbb{D}, f$ is quasiconformal in $E$.

A locally quasiconformal map $f$ has locally $L^{2}$-integrable formal generalized derivatives

$$
\partial_{z} f=\frac{1}{2}\left(\partial_{x} f-i \partial_{y} f\right), \quad \partial_{\bar{z}} f=\frac{1}{2}\left(\partial_{x} f+i \partial_{y} f\right) \quad(z=x+i y)
$$

and satisfies a Beltrami equation:

$$
\partial_{\bar{z}} f=\mu(z) \partial_{z} f, \quad\|\mu\|_{\infty} \leq 1 .
$$

Here $\mu_{f}(z)=\mu(z)=\frac{\partial_{z} f}{\partial_{z} f}$ is called the Beltrami coefficient or the complex dilatation of $f$. For any measurable subset $S$ of $\mathbb{D}$ we denote

$$
\|\mu\|_{S}=\operatorname{ess}_{\sup _{z \in S}}|\mu(z)|, \quad K_{S}[f]=\frac{1+\|\mu\|_{S}}{1-\|\mu\|_{S}}
$$

If $K[f]=K_{\mathbb{D}}[f]<\infty$, as usual, we say that $f$ is a $K$-quasiconformal map of $\mathbb{D}$ for any given $K \geq K[f]$.

Let $\left\{\gamma_{n}: \mathbb{D} \rightarrow \mathbb{C}\right\}$ be a sequence of maps on $\mathbb{D}$. If $\gamma_{n}$ converge to some map $\gamma: \mathbb{D} \rightarrow \mathbb{C}$ uniformly on any compact subset of $\mathbb{D}$ as $n \rightarrow \infty$, then we denote this by " $\gamma_{n} \rightrightarrows \gamma($ as $n \rightarrow \infty)$ ".

The main results of this paper are the following two theorems:

Theorem 1. Let $w_{n}=f_{n}: \mathbb{D} \rightarrow \mathbb{D}$ be a sequence of locally quasiconformal harmonic maps on the unit disk with respect to the Poincaré metric. Suppose the $\partial$-energy densities $\left|\partial w_{n}\right|^{2}$ of $f_{n}$ are uniformly bounded from below by a positive constant $\sigma$ and locally uniformly bounded from above. Then there is a subsequence $\left\{f_{n_{k}}\right\}$ of $\left\{f_{n}\right\}$ that locally uniformly converges on $\mathbb{D}$, and the limit function $w=f(z)$ of $\left\{f_{n_{k}}\right\}$ is either a locally quasiconformal harmonic map of the Poincaré disk or a constant. Especially, if $f(z)$ is not a constant, we can choose the subsequence $\left\{f_{n_{k}}\right\}$ 
such that the following conditions also hold: as $n_{k} \rightarrow \infty$,

$$
\begin{aligned}
& \left|\partial w_{n_{k}}\right|^{2} \rightrightarrows|\partial w|^{2}, \\
& \left|\bar{\partial} w_{n_{k}}\right|^{2} \rightrightarrows|\bar{\partial} w|^{2}, \\
& \mu_{f_{n_{k}}}(z) \rightrightarrows \mu(z)=\mu_{f}(z), \\
& \partial_{z} f_{n_{k}} \rightrightarrows \partial_{z} f, \\
& \partial_{\bar{z}} f_{n_{k}} \rightrightarrows \partial_{\bar{z}} f, \\
& \phi_{n_{k}} \rightrightarrows \phi=\phi_{f} .
\end{aligned}
$$

Theorem 1 tells us that certain boundedness of energy densities implies the normality of a sequence of harmonic maps as well as the normality of the corresponding sequence of Beltrami coefficients, and so on. Similar results have been obtained before under somewhat stronger assumptions. For example, Proposition 1.8 in [14 is achieved provided that the metrics $\rho\left(f_{n}\right)\left|\partial_{z} f_{n}\right|^{2}|d z|^{2}$ are complete on $\mathbb{D}$.

If the $f_{n}$ are $K$-quasiconformal harmonic maps, we have the following corollary.

Corollary 1. Let $w_{n}=f_{n}: \mathbb{D} \rightarrow \mathbb{D}$ be a sequence of $K$-quasiconformal harmonic maps on the Poincaré disk. Then there is a subsequence $\left\{f_{n_{k}}\right\}$ of $\left\{f_{n}\right\}$ that converges locally uniformly on $\mathbb{D}$ and the limit map $w=f(z)$ of the subsequence is either a K-quasiconformal harmonic map of the Poincaré disc or a constant. Moreover, if $f(z)$ is not a constant, then $\left\{f_{n_{k}}\right\}$ can be chosen such that 1.5 -(1.10) hold.

In [9], the consequence "the limit map $f$ is harmonic" of Corollary 1 plays a crucial role in the argument of the boundary value problem for harmonic maps related to the Schoen conjecture 11. But there is a gap in his proof (see pp. 2041-2044 of [9]). Our Corollary 1 just helps bridge the gap.

Recall that the universal Teichmüller space $T(\mathbb{D})$ is defined as the collection of all normalized quasisymmetric homeomorphisms $\varphi$ of $S^{1}=\partial \mathbb{D}$ onto itself. A map $\varphi: S^{1} \rightarrow S^{1}$ is said to be normalized quasisymmetric if it admits a quasiconformal extension to $\mathbb{D}$ and fixes the points $1,-1$, and $i$.

Definition 2. A quasiconformal map $f: \mathbb{D} \rightarrow \mathbb{D}$ is called asymptotically conformal if for every $\varepsilon>0$ there is a compact set $E$ in $\mathbb{D}$ such that $f$ is $(1+\varepsilon)$-quasiconformal on $\mathbb{D} \backslash E$.

Let $T_{0}(\mathbb{D})$ be the set of all $\varphi \in T(\mathbb{D})$ that admit an asymptotically conformal extension to $\mathbb{D}$. $T_{0}(\mathbb{D})$ is a closed subspace of the universal Teichmüller space [6].

Let $q: S^{1} \rightarrow S^{1}$ be a quasisymmetric map of the unit circle. The map $q$ is said to be symmetric (see [6], [13]) if

$$
\lim _{t \rightarrow 0} \frac{q\left(e^{i\left(t+t_{0}\right)}\right)-q\left(e^{i t_{0}}\right)}{q\left(e^{i t_{0}}\right)-q\left(e^{i\left(t_{0}-t\right)}\right)}=1,
$$

uniformly in $t$ for all points $z_{0}=e^{i t_{0}} \in S^{1}$.

Strebel [13] and Fehlmann [5] showed respectively that a normalized quasisymmetric map $\varphi \in T_{0}(\mathbb{D})$ if and only if $\varphi$ is a symmetric map of the unit circle.

Recently, Earle, Marković and Saric's joint work [4 indicates that the barycentric extension (or Douady-Earle extension, see [3]) of every $\varphi \in T_{0}(\mathbb{D})$ is an asymptotic hyperbolic isometry. Following their idea, as an application of Corollary 1 we claim 
that the quasiconformal harmonic extension of $\varphi \in T_{0}(\mathbb{D})$ is also an asymptotic hyperbolic isometry. To be precise, we have

Theorem 2. Every $\varphi \in T_{0}(\mathbb{D})$ admits a unique quasiconformal harmonic extension to $\mathbb{D}$ that is an asymptotic hyperbolic isometry.

\section{Some LEMMAS}

We have restricted ourselves to the case that a harmonic map should be a $C^{2}$ diffeomorphism. So for a harmonic map $w,|\partial w|^{2}>0$ and $J(w)>0$, and hence the function

$$
u=\frac{1}{2} \log |\partial w|^{2}
$$

is well defined in $\mathbb{D}$. The following Bochner equation can be deduced from equation (1.2):

$$
\Delta_{\rho} u=e^{2 u}-\|\Phi\|^{2} e^{-2 u}-1,
$$

where

$$
\|\Phi\|=\rho^{-1}(z)|\phi(z)|, \quad \phi(z)=\rho(w) w_{z} \bar{w}_{z} .
$$

We include two main results from [16] here as Lemma 2.1 and Lemma 2.2 .

Lemma 2.1. Let $w=f(z)$ be a harmonic map of $\mathbb{D}$ onto itself. Then $w=f(z)$ is a quasiconformal map if and only if the Bers norm of its Hopf differential $\phi(z)=$ $\rho(w) w_{z} \bar{w}_{z}=\rho(w) w_{z} \bar{w}_{z}$ is bounded, i.e.,

$$
\|\phi\|_{B}=\sup _{z \in \mathbb{D}}\left|\rho^{-1}(z) \phi(z)\right|<\infty .
$$

Lemma 2.2. Let $w=f(z)$ be a quasiconformal harmonic map of $\mathbb{D}$ onto itself. Then we have

$$
1 \leq|\partial w|^{2}=\frac{\rho(f(z))}{\rho(z)}\left|f_{z}(z)\right|^{2} \leq \frac{1}{2}\left(1+\sqrt{1+4\|\phi\|_{B}^{2}}\right), \forall z \in \mathbb{D},
$$

where $\phi$ is the Hopf differential of $w=f(z)$.

From Lemma [2.2, we can easily deduce the following corollary:

Corollary 2.1. Let $w=f(z)$ be a quasiconformal harmonic map of $\mathbb{D}$ onto itself. Then

$$
\|\phi\|_{B} \leq \frac{K[f]-1}{2}
$$

and

$$
1 \leq|\partial w|^{2} \leq \frac{K[f]+1}{2} .
$$

Proof. Since $\mu_{f}(z)=\frac{\rho^{-1}(z) \overline{\phi(z)}}{|\partial w|^{2}}$, we have

$$
\left\|\mu_{f}\right\|_{\infty} \geq \frac{2\|\phi\|_{B}}{1+\sqrt{1+4|| \phi \|_{B}^{2}}} \geq \frac{2\|\phi\|_{B}}{2+2\|\phi\| \|_{B}}=\frac{\|\phi\|_{B}}{1+\|\phi\|_{B}} .
$$

Thus, (2.5) and (2.6) follow from (2.4) and (2.7). 
To prove our main results, we have to estimate the gradient of the energy density of a harmonic map. The estimate is very important in the proof of Theorem [

Lemma 2.3. Suppose $w$ is a harmonic diffeomorphism on $\mathbb{D}$ with $|\partial w|^{2}>\sigma$, where $\sigma$ is a positive constant. Let $\Phi=\phi d z^{2}$ be the Hopf differential of $w$. Set

$$
\mathbb{D}_{r}=\{|z|<r<1\}
$$

and

$$
m_{r}=\sup _{z \in \mathbb{D}_{\frac{1+r}{2}}}|\partial w|^{2}
$$

Then

$$
\sup _{z \in \mathbb{D}_{r}}\left|\nabla\left(\log |\partial w|^{2}\right)\right| \leq C m_{r}
$$

for some constant $C$ depending only on $r$, where $\nabla$ is the gradient in the Poincaré disk $\mathbb{D}$.

Proof. Let $u=\frac{1}{2} \log |\partial w|^{2}$. Since $w$ is a locally quasiconformal diffeomorphism, $|\partial w|^{2}>\sigma$ everywhere and hence $u$ is well-defined and smooth in $\mathbb{D}$. By (2.1) we have

$$
\sup _{z \in \mathbb{D}_{r}} \rho^{-1}(z)\left|\Delta_{0} u\right| \leq C_{1} m_{r}
$$

where $C_{1}$ is a constant depending only on $r$. Let $z_{0}=x_{0}+i y_{0} \in \mathbb{D}_{r}$. Set $\delta=\frac{1-\left|z_{0}\right|}{4}$ and $B_{\delta}\left(z_{0}\right)=\left\{z:\left|z-z_{0}\right|<\delta\right\}$. Then $B_{2 \delta}\left(z_{0}\right)=\left\{z:\left|z-z_{0}\right|<2 \delta\right\}$ is contained in $\mathbb{D}_{\frac{1+r}{2}}$. Applying the estimate on p. 70 in [7] to $B_{\delta}\left(z_{0}\right)$ and $B_{2 \delta}\left(z_{0}\right)$, we see that there is an absolute constant $C_{2}$ such that

$$
\left(1-\left|z_{0}\right|^{2}\right)\left|\nabla_{0} u\right|\left(z_{0}\right) \leq C_{2}\left(\sup _{B_{2 \delta}}|u|+m_{r}\right),
$$

where $\nabla_{0}$ is the usual Euclidean gradient. Using the fact that $\log (1+t) \leq t$ for $t \geq 0$, we have

$$
\sup _{B_{2 \delta}}|u| \leq \frac{1}{2}\left(\log \frac{m_{r}}{\sigma}+|\log \sigma|\right) \leq \frac{m_{r}}{\sigma}+\frac{1}{2}|\log \sigma| \leq C_{3} m_{r},
$$

where $C_{3}$ depends only on $r$. Thus the lemma follows.

We also need the following classical results on good approximation to a quasiconformal map.

Lemma 2.4 ([8]). Let $w_{n}, n=1,2, \ldots$, be a sequence of $K$-quasiconformal maps of $G$ that converges to a quasiconformal map $w$ with complex dilatation $\mu$, uniformly on compact subsets of $G$. If the complex dilatations $\mu_{n}(z)$ of $w_{n}$ tend to limit $\mu_{\infty}(z)$ almost everywhere in $G$, then $w_{n}$ is a good approximation to $w$, i.e., $\mu_{\infty}(z)=\mu(z)$ almost everywhere in $G$. Moreover, there exists a subsequence $\left\{w_{n_{k}}\right\}$ of $\left\{w_{n}\right\}$ such that $\partial_{z} w_{n_{k}} \rightarrow \partial_{z} w, \partial_{\bar{z}} w_{n_{k}} \rightarrow \partial_{\bar{z}} w\left(\right.$ as $\left.n_{k} \rightarrow \infty\right)$ almost everywhere in $G$.

Finally, we introduce

Definition 3. Let $\mu \in L^{\infty}(\mathbb{D})$ be a Beltrami coefficient of a quasiconformal map of $\mathbb{D}$. We say that $\mu$ vanishes at infinity on $\mathbb{D}$ if and only if for every $\varepsilon>0$ there is a compact set $E$ in $\mathbb{D}$ such that $\|\mu\|_{\mathbb{D} \backslash E}$ is less than $\varepsilon$. 
Let $d_{\mathbb{D}}(z, w)=2 \tanh ^{-1}\left|\frac{z-w}{1-\bar{w} z}\right|$ denote the hyperbolic distance of $z, w \in \mathbb{D}$. For any $a \in \mathbb{D}$ and $r>0$, let $\bar{B}(a, r)$ be the closed hyperbolic ball of radius $r$ and center $a$.

Let $\left\{p_{n}\right\}$ be a sequence in $\mathbb{D}$. We say that $p_{n}$ approaches infinity in $\mathbb{D}$ and write $p_{n} \rightarrow \infty$ if and only if $\lim _{n \rightarrow \infty} d_{\mathbb{D}}\left(p, p_{n}\right)=\infty$ for some, hence all, $p \in \mathbb{D}$. With these definitions the following lemma was proved in [4].

Lemma 2.5. Let $\mu$ be a Beltrami coefficient, and let $r$ be a positive real number. Then $\mu$ vanishes at infinity on $\mathbb{D}$ if and only if $\lim _{n \rightarrow \infty}\|\mu\|_{\bar{B}\left(z_{n}, r\right)}=0$ for every sequence $\left\{z_{n}\right\}$ in $\mathbb{D}$ such that $z_{n} \rightarrow \infty$.

\section{Proof of Theorem 1}

In the proof, we shall apply the diagonal trick to choose a convergent subsequence from some sequence many times. For simplicity and without loss of generality, unless otherwise stated, we still use the original sequence to represent the subsequence after passing to a subsequence if necessary.

Suppose $w_{n}=f_{n}: \mathbb{D} \rightarrow \mathbb{D}$ is a sequence of harmonic diffeomorphisms of $\mathbb{D}$ onto itself satisfying the conditions in Theorem 1. Let $\phi_{n}$ be the Hopf differential of $f_{n}$, i.e., $\phi_{n}=\rho\left(f_{n}\right) \partial_{z} f_{n} \partial_{z} \overline{f_{n}}$. Then $\phi_{n}$ is holomorphic in $\mathbb{D}$. Suppose $N(r)=$ $\sup _{n \in \mathbb{N}} \sup _{z \in \mathbb{D}_{r}}\left|\partial w_{n}\right|^{2}$. Then, in $\mathbb{D}_{r}$,

$$
\left|\phi_{n}(z)\right|=\rho\left(f_{n}\right)\left|\partial_{z} f_{n}\right|\left|\partial_{\bar{z}} f_{n}\right| \leq \rho(z)\left|\partial w_{n}\right|^{2} \leq M(r) N(r),
$$

where $M(r)=4 /\left(1-r^{2}\right)^{2}$. This implies that $\left\{\phi_{n}\right\}$ is normal in $\mathbb{D}$.

Next we show that $\left\{\left|\partial w_{n}\right|^{2}\right\}$ is normal in $\mathbb{D}$. It is sufficient to prove that $\left\{\left|\partial w_{n}\right|^{2}\right\}$ is equicontinuous in $\mathbb{D}$ by virtue of the classical theory of normal families. Since $\left.\left|\nabla_{0}\right| \partial w_{n}\right|^{2}|=| \partial_{z}\left|\partial w_{n}\right|^{2}|+| \partial_{\bar{z}}\left|\partial w_{n}\right|^{2} \mid$, we have

$$
\left|\nabla\left(\log \left|\partial w_{n}\right|^{2}\right)\right|=\frac{\left.|\nabla| \partial w_{n}\right|^{2} \mid}{\left|\partial w_{n}\right|^{2}}=\frac{\left(1-|z|^{2}\right)\left(\left.\left|\partial_{z}\right| \partial w_{n}\right|^{2}|+| \partial_{\bar{z}}\left|\partial w_{n}\right|^{2} \mid\right)}{2\left|\partial w_{n}\right|^{2}} .
$$

Since $\left|\partial w_{n}\right|^{2}$ is bounded from below by a positive constant $\sigma$ and is locally uniformly bounded from above, we see that $\left.\left|\partial_{z}\right| \partial w_{n}\right|^{2} \mid$ and $\left.\left|\partial_{\bar{z}}\right| \partial w_{n}\right|^{2} \mid$ are uniformly bounded in $\mathbb{D}_{r}$ from Lemma 2.3. This indicates that $\left\{\left|\partial w_{n}\right|^{2}\right\}$ is equicontinuous.

Note that $\mu_{f_{n}}=\frac{\rho^{-1}(z) \overline{\phi_{n}(z)}}{\left|\partial w_{n}\right|^{2}}$. We know that $\left\{\mu_{f_{n}}\right\}$ is normal from the normality of $\left\{\left|\partial w_{n}\right|^{2}\right\}$ and $\left\{\phi_{n}\right\}$. So

$$
\left\|\mu_{f_{n}}\right\|_{\mathbb{D}_{r}} \leq s(r)<1, \quad K_{\mathbb{D}_{r}}\left[f_{n}\right]=\frac{1+\left\|\mu_{f_{n}}\right\|_{\mathbb{D}_{r}}}{1-\left\|\mu_{f_{n}}\right\|_{\mathbb{D}_{r}}} \leq \frac{1+s(r)}{1-s(r)}
$$

where $s(r)$ is a constant only depending on $r$. This implies that $\left\{f_{n}\right\}$ is normal in $\mathbb{D}$ and so is $\left\{\rho\left(f_{n}\right)=\frac{4}{\left(1-\left|f_{n}\right|^{2}\right)^{2}}\right\}$ as well. Suppose $f$ is the limit map which is not a constant. Obviously, the map $f$ is a locally quasiconformal map with $K_{\mathbb{D}_{r}}[f] \leq(1+s(r)) /(1-s(r))$.

Furthermore, we can conclude from Lemma 2.4 that (1.7) holds.

Next, we prove that $f$ is harmonic in $\mathbb{D}$. For this, we only need to show that (1.8) and (1.9) hold. 
First, we show that

$$
\partial_{z} f_{n} \rightrightarrows \partial_{z} f, \quad \text { as } n \rightarrow \infty .
$$

By computation, we have

$$
0=\partial_{\bar{z}} \phi_{n}=\partial_{\bar{z}} \rho\left(f_{n}\right) \partial_{z} f_{n} \partial_{z} \overline{f_{n}}+\rho\left(f_{n}\right)\left(\partial_{z} \partial_{\bar{z}} f_{n} \partial_{z} \overline{f_{n}}+\partial_{z} f_{n} \partial_{z} \partial_{\bar{z}} \overline{f_{n}}\right) .
$$

It follows from (3.4) that

$$
\begin{aligned}
\left|\partial_{\bar{z}} \rho\left(f_{n}\right) \partial_{z} f_{n} \partial_{z} \overline{f_{n}}\right| & \geq \rho\left(f_{n}\right)\left|\partial_{z} \partial_{\bar{z}} f_{n} \| \partial_{z} f_{n}\right|\left(1-\| \mu_{f_{n}}||_{\mathbb{D}_{r}}\right) \\
& \geq \rho\left(f_{n}\right)\left|\partial_{z} \partial_{\bar{z}} f_{n} \| \partial_{z} f_{n}\right|(1-s(r)), \quad z \in \mathbb{D}_{r} .
\end{aligned}
$$

Rewrite the above inequality in the following form:

$$
\left|\partial_{\bar{z}} \partial_{z} f_{n}\right| \leq \frac{\left|\partial_{\bar{z}} \rho\left(f_{n}\right)\right|\left|\partial_{\bar{z}} f_{n}\right|}{(1-s(r)) \rho\left(f_{n}\right)}
$$

A simple computation shows

$$
\left|\partial_{\bar{z}} \rho\left(f_{n}\right) \partial_{\bar{z}} f_{n}\right|=8 \frac{\left|\overline{f_{n}}\left(\partial_{\bar{z}} f_{n}\right)^{2}+f_{n} \overline{\partial_{z} f_{n}} \partial_{\bar{z}} f_{n}\right|}{\left(1-\left|f_{n}\right|^{2}\right)^{3}} \leq 8 \frac{\left|f_{n}\right|\left|\partial_{\bar{z}} f_{n}\right|^{2}}{\left(1-\left|f_{n}\right|^{2}\right)^{3}}+2 \frac{\left|f_{n}\right|\left|\phi_{n}\right|}{1-\left|f_{n}\right|^{2}} .
$$

On the other hand,

$$
\begin{aligned}
\partial_{z}\left|\partial w_{n}\right|^{2} & =\partial_{z}\left(\frac{\rho\left(f_{n}\right)}{\rho(z)} \partial_{z} f_{n} \partial_{\bar{z}} \overline{f_{n}}\right) \\
& =\partial_{z}\left(\frac{\rho\left(f_{n}\right)}{\rho(z)}\right) \partial_{z} f_{n} \partial_{\bar{z}} \overline{f_{n}}+\frac{\rho\left(f_{n}\right)}{\rho(z)}\left(\partial_{\bar{z}} \overline{f_{n}} \partial_{z} \partial_{z} f_{n}+\partial_{z} f_{n} \partial_{z} \partial_{\bar{z}} \overline{f_{n}}\right) .
\end{aligned}
$$

Then

$$
\left|\partial_{z} \partial_{z} f_{n}\right| \leq \frac{\rho(z)}{\rho\left(f_{n}\right)\left|\partial_{z} f_{n}\right|}\left[\left.\left.\left|\partial_{z}\right| \partial w_{n}\right|^{2}|+| \partial_{z}\left(\frac{\rho\left(f_{n}\right)}{\rho(z)}\right)|| \partial_{z} f_{n}\right|^{2}\right]+\left|\partial_{z} \partial_{\bar{z}} f_{n}\right| .
$$

In addition,

$$
\begin{aligned}
& \left|\partial_{z}\left(\frac{\rho\left(f_{n}\right)}{\rho(z)}\right)\right|=\left|\partial_{z} \frac{\left(1-|z|^{2}\right)^{2}}{\left(1-\left|f_{n}\right|^{2}\right)^{2}}\right| \\
& =\frac{2\left(1-|z|^{2}\right)}{\left(1-\left|f_{n}\right|^{2}\right)^{3}}\left|\left(1-\left|f_{n}\right|^{2}\right) \bar{z}-\left(1-|z|^{2}\right)\left(\overline{f_{n}} \partial_{z} f_{n}+f_{n} \partial_{z} \overline{f_{n}}\right)\right| \\
& \leq \frac{2\left(1-|z|^{2}\right)}{\left(1-\left|f_{n}\right|^{2}\right)^{3}}\left[\left(1-\left|f_{n}\right|^{2}\right)+\left|f_{n}\right|\left(1-|z|^{2}\right)\left(\left|\partial_{z} f_{n}\right|+\left|\partial_{\bar{z}} f_{n}\right|\right)\right] .
\end{aligned}
$$

Since $\left\{f_{n}\right\}$ is normal and $\left|\partial w_{n}\right|^{2},\left|\bar{\partial} w_{n}\right|^{2}$ are uniformly bounded in $\mathbb{D}_{r}$, combining (3.5) (3.6) (3.7) and (3.8), one can easily verify that $\left|\partial_{\bar{z}} \partial_{z} f_{n}\right|$ and $\left|\partial_{z} \partial_{z} f_{n}\right|$ are uniformly bounded in $\mathbb{D}_{r}$. Therefore, $\left\{\partial_{z} f_{n}\right\}$ is equicontinuous in $\mathbb{D}$. Consequently, $\left\{\partial_{z} f_{n}\right\}$ is normal.

Note that $\partial_{\bar{z}} f_{n}=\mu_{f_{n}} \partial_{z} f_{n}$. We can conclude that $\left\{\partial_{\bar{z}} f_{n}\right\}$ is also normal in $\mathbb{D}$. Further application of Lemma 2.4 offers (1.8) and (1.9). In the meantime, for $\left|\partial w_{n}\right|^{2}=\frac{\rho\left(f_{n}\right)}{\rho(z)}\left|\partial_{z} f_{n}\right|^{2}$ and $\left|\bar{\partial} w_{n}\right|^{2}=\frac{\rho\left(f_{n}\right)}{\rho(z)}\left|\partial_{\bar{z}} f_{n}\right|^{2}$, (1.5) and (1.6) hold.

Finally, it follows readily that $\phi_{n} \rightrightarrows \phi_{f}($ as $n \rightarrow \infty)$ from $\phi_{n}=\rho\left(f_{n}\right) \partial_{z} f_{n} \partial_{z} \overline{f_{n}}$. Therefore, $\phi_{f}$ is holomorphic in $\mathbb{D}$, and hence $f$ is a harmonic homeomorphism of $\mathbb{D}$. This completes the proof of Theorem 1 .

Now, Corollary 1 derives from Theorem 1 and Corollary 2.1 immediately. 


\section{Proof of Theorem 2}

The following lemma is implied in Theorem 3.1 of [10]:

Lemma 4.1. Every $\varphi \in T_{0}(\mathbb{D})$ has a unique quasiconformal harmonic extension $H_{\varphi}$ to $\mathbb{D}$, and the Hopf differential $\phi d z^{2}$ of $H_{\varphi}$ satisfies

$$
\begin{aligned}
\phi d z^{2} & \in B Q D_{0}(\mathbb{D}) \\
& =\left\{\psi(z) d z^{2} \text { is holomorphic in } \mathbb{D}: \lim _{r \rightarrow 0} \sup _{1-r<|z|<1} \rho^{-1}(z)|\psi(z)|=0\right\} .
\end{aligned}
$$

Actually, to prove Theorem 2, we only need to show the following precise form.

Lemma 4.2. For any given $\varphi \in T_{0}(\mathbb{D})$, let $w=H_{\varphi}$ denote the quasiconformal harmonic extension of $\varphi$ to $\mathbb{D}$ with respect to the Poincaré metric. Then for any $\varepsilon>0$ there is a compact set $E \subset \mathbb{D}$ such that

$$
(1+\varepsilon)^{-1} d_{\mathbb{D}}(x, y) \leq d_{\mathbb{D}}\left(H_{\varphi}(x), H_{\varphi}(y)\right) \leq(1+\varepsilon) d_{\mathbb{D}}(x, y)
$$

for all $(x, y) \in(\mathbb{D} \times \mathbb{D}) \backslash(E \times E)$.

Before the proof of Lemma 4.2. We apply Corollary 1 to prove an infinitesimal version of Lemma 4.2 .

Lemma 4.3. Let $\varphi \in T_{0}(\mathbb{D})$, and let $\left(H_{\varphi}\right)_{*}$ be the map of tangent bundles induced by $H_{\varphi}$. Let $\left\{z_{n}\right\}$ be a sequence of points in $\mathbb{D}$ such that $z_{n} \rightarrow \infty$, and let $v_{n}$ be a tangent vector of hyperbolic length one at $z_{n}$ for each $n$. Then

$$
\lim _{n \rightarrow \infty}\left\|\left(H_{\varphi}\right)_{*} v_{n}\right\|_{\mathbb{D}}=1,
$$

where $\|\cdot\|_{\mathbb{D}}$ denotes the hyperbolic length of some tangent vector to $\mathbb{D}$.

Proof. Let $\mu$ be the complex dilatation of $H_{\varphi}$. For each $n$ choose an automorphism $g_{n}$ of $\mathbb{D}$ such that $g_{n}$ maps 0 to $z_{n}$, and set $\mu_{n}=g_{n}^{*}(\mu)=\left(\mu \circ g_{n}\right) \overline{g_{n}^{\prime}} / g_{n}^{\prime}$. Since $\mu(z)=\frac{\rho^{-1}(z) \overline{\phi(z)}}{|\partial w|^{2}}$, Lemma 4.1 implies that $\mu$ vanishes at infinity on $\mathbb{D}$. We conclude from Lemma 2.5 and the definition of $\mu_{n}$ that

$$
\lim _{n \rightarrow \infty}\left\|\mu_{n}\right\|_{\bar{B}(0, r)}=\lim _{n \rightarrow \infty}\|\mu\|_{\bar{B}\left(z_{n}, r\right)}=0, \quad \forall r>0 .
$$

For each $n$ there is an automorphism $\gamma_{n}$ of $\mathbb{D}$ such that

$$
\varphi_{n}=\left.\gamma_{n} \circ H_{\varphi} \circ g_{n}\right|_{\partial \mathbb{D}} \in T_{0}(\mathbb{D}) .
$$

Since the harmonicity is conformally invariant, we have $H_{\varphi_{n}}=\gamma_{n} \circ H_{\varphi} \circ g_{n}$ and hence $H_{\varphi_{n}}$ are all $K$-quasiconformal harmonic, with $K=\left(1+\|\mu\|_{\infty}\right) /\left(1-\|\mu\|_{\infty}\right)$ independent of $n$, and $\mu_{n}$ is just the complex dilatation of $H_{\varphi_{n}}$. It follows readily from Corollary 1 and (4.4), by passing to a subsequence if necessary, that $H_{\varphi_{n}}$ converges uniformly on compact sets in $\mathbb{D}$ to the identity map as $n \rightarrow \infty$. Moreover, $\partial_{z} H_{\varphi_{n}} \rightarrow 1$ and $\partial_{\bar{z}} H_{\varphi_{n}} \rightarrow 0$ as $n \rightarrow \infty$, uniformly on compact sets in $\mathbb{D}$. Thus we have proved that for any $\delta>0$ there is an integer $N$ such that

$$
(1+\delta)^{-1} \leq\left\|\left(H_{\varphi_{n}}\right)_{*}(v)\right\|_{\mathbb{D}} \leq 1+\delta
$$

whenever $n>N$ and $v$ is a unit tangent vector to $\mathbb{D}$ at the point 0 . 
Because all automorphisms of $\mathbb{D}$ are hyperbolic isometries, we have

$$
(1+\delta)^{-1} \leq\left\|\left(H_{\varphi}\right)_{*}\left(v_{n}\right)\right\|_{\mathbb{D}}=\left\|\left(H_{\varphi_{n}}\right)_{*} v\right\|_{\mathbb{D}} \leq 1+\delta
$$

whenever $n>N$ and $v_{n}$ is a unit tangent vector to $\mathbb{D}$ at the point $z_{n}$.

Remark 1. The above proof imitates that of Lemma 13.2 of [4].

The proof of Lemma 4.2 almost takes word by word from that of Lemma 13.1 in [4]. For completeness and for convenience of the reader, we reproduce the proof as follows.

We denote the hyperbolic length of a smooth curve $c(t)$ in $\mathbb{D}$ by $L(c)$.

Let $\varepsilon>0$ be given. We shall derive the desired inequality (4.2) from Lemma 4.3 By that lemma we can choose a compact set $E_{0} \subset \mathbb{D}$ so that

$$
\left(1+\frac{\varepsilon}{2}\right)^{-1}\|v\|_{\mathbb{D}} \leq\left\|w_{*}(v)\right\|_{\mathbb{D}} \leq\left(1+\frac{\varepsilon}{2}\right)\|v\|_{\mathbb{D}}
$$

whenever $v$ is a tangent vector to $\mathbb{D}$ at a point of $\mathbb{D} \backslash E_{0}$.

Let $R$ be the hyperbolic diameter of $w\left(E_{0}\right)$. We claim that if $x \in \mathbb{D}$ satisfies the condition $d_{\mathbb{D}}\left(x, E_{0}\right)>2 R / \varepsilon$, then

$$
d_{\mathbb{D}}(w(x), w(y)) \leq(1+\varepsilon) d_{\mathbb{D}}(x, y)
$$

for all $y \in \mathbb{D}$.

To prove this claim, we consider a distance minimizing geodesic $c:[0,1] \rightarrow \mathbb{D}$ that joins $x=c(0)$ to $y=c(1)$.

If $c(t) \in \mathbb{D} \backslash E_{0}$ for all $t \in[0,1]$, then inequality (4.7) gives

$$
L(w \circ c) \leq\left(1+\frac{\varepsilon}{2}\right) L(c)=\left(1+\frac{\varepsilon}{2}\right) d_{\mathbb{D}}(x, y) .
$$

Since the curve $w \circ c$ joins $w(x)$ to $w(y)$, we obtain

$$
d_{\mathbb{D}}(w(x), w(y)) \leq\left(1+\frac{\varepsilon}{2}\right) d_{\mathbb{D}}(x, y) .
$$

If $c(t) \in E_{0}$ for some $t \in[0,1]$, set

$$
a=\min \left\{t \in[0,1]: c(t) \in E_{0}\right\} \quad \text { and } \quad b=\max \left\{t \in[0,1]: c(t) \in E_{0}\right\} .
$$

The curves $c:[0, a] \rightarrow \mathbb{D}$ and $c:[b, 1] \rightarrow \mathbb{D}$ are distance minimizing geodesics, and the sum of their lengths is at most $d_{\mathbb{D}}(x, y)$. Since (apart from their endpoints) they avoid $E_{0}$, the reasoning that gave (4.9) yields

$$
d_{\mathbb{D}}(w(x), w(c(a)))+d_{\mathbb{D}}(w(c(b)), w(y)) \leq\left(1+\frac{\varepsilon}{2}\right) d_{\mathbb{D}}(x, y) .
$$

Since both $c(a)$ and $c(b)$ belong to $E_{0}$, the definition of $R$ and our condition on $x$ give

$$
d_{\mathbb{D}}(w(c(a)), w(c(b))) \leq R<\frac{\varepsilon}{2} d_{\mathbb{D}}\left(x, E_{0}\right) \leq \frac{\varepsilon}{2} d_{\mathbb{D}}(x, c(a)) \leq \frac{\varepsilon}{2} d_{\mathbb{D}}(x, y) .
$$

Adding these inequalities and applying the triangle inequality, we obtain (4.8), proving our claim.

By the same reasoning, we have

$$
d_{\mathbb{D}}(x, y) \leq(1+\varepsilon) d_{\mathbb{D}}(w(x), w(y)), \forall y \in \mathbb{D},
$$

if $x \in \mathbb{D}$ satisfies the condition $d_{\mathbb{D}}\left(w(x), w\left(E_{0}\right)\right)>2 D / \varepsilon$, where $D$ is the hyperbolic diameter of $E_{0}$. We will not go into detail. 
We conclude that (4.2) holds unless both $x$ and $y$ belong to the compact set

$$
E=\left\{x \in \mathbb{D}: d_{\mathbb{D}}\left(x, E_{0}\right) \leq 2 R / \varepsilon \text { or } d_{\mathbb{D}}\left(w(x), w\left(E_{0}\right)\right) \leq 2 D / \varepsilon\right\} .
$$

If $\varphi \in T(\mathbb{D})$ admits a quasiconformal harmonic extension $H_{\varphi}$ to $\mathbb{D}$, then $H_{\varphi}$ is a hyperbolic quasi-isometry on $\mathbb{D}[16]$. Our Lemma 4.2 gives a somewhat more subtle form for $\varphi \in T_{0}(\mathbb{D})$.

Remark 2. Another further application of our Corollary 1 1 shows [18] that the harmonic embedding map from the Bers-norm bounded Hopf differentials space to the Teichmüller space $T(X)$ induces an embedding from an appropriate quotient Banach space into the asymptotic Teichmüller space $A T(X)$, where $X$ is a hyperbolic Riemann surface. In addition, if the Schoen conjecture is true, i.e., every $\varphi \in T(\mathbb{D})$ has a quasiconformal harmonic extension to $\mathbb{D}$, then our Theorem 2 can be generalized to any hyperbolic Riemann surface.

Remark 3. If the quasiconformal map $f$ is an asymptotic hyperbolic isometry on $\mathbb{D}$, then $\left.f\right|_{\partial \mathbb{D}}$ is a symmetric map of the unit circle.

\section{ACKNOWLEDGEMENT}

The author would like to express great gratitude to the referee for valuable comments and suggestions. Thanks are also due to Professor Li Zhong and Professor $\mathrm{Wu}$ Shengjian for their continuous encouragement and support.

\section{REFERENCES}

[1] I. Anić, V. Marković, and M. Mateljiević, Uniformly bounded maximal $\phi$-disks, Bers space and harmonic maps, Proc. Amer. Math. Soc., 128 (2000), 2947-2956. MR 2000m:30054

[2] S. Y. Cheng, L. F. Tam, and Tom Y. H. Wan, Harmonic maps with finite total energy, Proc. Amer. Math. Soc., 124 (1996), 275-284. MR 96d:58031

[3] A. Douady and C. J. Earle, Conformally natural extension of homeomorphisms of the circle, Acta Math., 157 (1986), 23-48. MR 87j:30041

[4] C. J. Earle, V. Marković, and D. Saric, Barycentric extension and the Bers embedding for asymptotic Teichmüller space, Contemp. Math. 311, Amer. Math. Soc., Providence, RI, 2002, pp. 87-105. MR 2003i:30072

[5] R. Fehlmann, Ueber extremale quasikonforme Abbildungen, Comment. Math. Helv., 56 (1981), 558-580. MR 83e:30024

[6] F. P. Gardiner and D. P. Sullivan, Symmetric structures on a closed curve, Amer. J. Math., 114 (1992), 683-736. MR 95h:30020

[7] D. Gilbarg and N. S. Trudinger, Elliptic partial differential equations of second order, 2nd edition, Springer-Verlag, 1983. MR 86c:35035

[8] O. Lehto and K. I. Virtanen, Quasiconformal mappings in the plane, Springer-Verlag, Berlin and New York, 1973. MR 49:9202

[9] Z. Li, On the boundary value problem for harmonic maps of the Poincaré disc, Chinese Sci. Bull., 42 (1997), 2025-2045. MR 2000b:30060

[10] V. Marković, Harmonic diffeomorphisms of noncompact surfaces and Teichmüller spaces, J. London Math. Soc. (2) 65 (2002), 103-114. MR 2002k:32015

[11] R. Schoen, The role of harmonic mappings in rigidity and deformation problems, Collection: Complex Geometry (Osaka, 1990), Lecture Notes in Pure and Applied Mathematics, Vol. 143, Dekker, New York, 1993, pp. 179-200. MR 94g:58055

[12] R. Schoen and S. T. Yau, On univalent harmonic maps between surfaces, Invent. Math., 44 (1978), 265-278. MR 57:17706

[13] K. Strebel, On the existence of extremal Teichmüller mappings, J. Analyse Math., 30 (1976), 464-480. MR 55:12912

[14] L. F. Tam and Tom Y. H. Wan, Harmonic diffeomorphisms into Cartan-Hadamard surfaces with prescribed Hopf differentials, Comm. Anal. Geom., 2 (1994), 593-625. MR 96m:58057 
[15] L. F. Tam and Tom Y. H. Wan, Quasi-conformal harmonic diffeomorphism and the universal Teichmüller space, J. Differential Geom. 42 (1995), 368-410. MR 96j:32024

[16] Tom Y. H. Wan, Constant mean curvature surface, harmonic maps and universal Teichmüller space, J. Differential Geom., 35 (1992), 643-657. MR 94a:58053

[17] M. Wolf, The Teichmüller theory of harmonic maps, J. Differential Geom. 29 (1989), 449-479. MR 90h:58023

[18] G. W. Yao, Harmonic maps and asymptotic Teichmüller space, preprint (2001).

School of Mathematical Sciences, Peking University, Beijing, 100871, People's RePUBLIC OF CHINA

E-mail address: wallgreat@lycos.com

Current address: Institute of Mathematics, Academy of Mathematics and Systems Science, Chinese Academy of Sciences, Beijing, 100080, People's Republic of China 